\title{
Cardiac-specific Overexpression of Phospholamban Alters Calcium Kinetics and Resultant Cardiomyocyte Mechanics in Transgenic Mice
}

\author{
Vivek J. Kadambi, ${ }^{*}$ Sathivel Ponniah,, Judy M. Harrer, ${ }^{*}$ Brian D. Hoit, ${ }^{\ddagger}$ Gerald W. Dorn II, ${ }^{\ddagger}$ Richard A. Walsh, ${ }^{\ddagger}$ \\ and Evangelia G. Kranias* \\ $*$ Departments of Pharmacology and Cell Biophysics, and ${ }^{\ddagger}$ Division of Cardiology, University of Cincinnati, College of Medicine, \\ Cincinnati, Ohio 45267
}

\begin{abstract}
Phospholamban is the regulator of the cardiac sarcoplasmic reticulum (SR) $\mathrm{Ca}^{2+}$-ATPase activity and an important modulator of basal contractility in the heart. To determine whether all the SR $\mathrm{Ca}^{2+}$-ATPase enzymes are subject to regulation by phospholamban in vivo, transgenic mice were generated which overexpressed phospholamban in the heart, driven by the cardiac-specific $\alpha$-myosin heavy chain promoter. Quantitative immunoblotting revealed a twofold increase in the phospholamban protein levels in transgenic hearts compared to wild type littermate hearts. The transgenic mice showed no phenotypic alterations and no changes in heart/body weight, heart/lung weight, and cardiomyocyte size. Isolated unloaded cardiac myocytes from transgenic mice exhibited diminished shortening fraction $(63 \%)$ and decreased rates of shortening $(64 \%)$ and relengthening (55\%) compared to wild type (100\%) cardiomyocytes. The decreases in contractile parameters of transgenic cardiomyocytes reflected decreases in the amplitude $(83 \%)$ of the $\mathrm{Ca}^{2+}$ signal and prolongation $(131 \%)$ in the time for decay of the $\mathrm{Ca}^{2+}$ signal, which was associated with a decrease in the apparent affinity of the SR Ca${ }^{2+}$-ATPase for $\mathrm{Ca}^{2+}(56 \%)$, compared to wild type $(100 \%)$ cardiomyocytes. In vivo analysis of left ventricular systolic function using M mode and pulsed-wave Doppler echocardiography revealed decreases in fractional shortening (79\%) and the normalized mean velocity of circumferential shortening $(67 \%)$ in transgenic mice compared to wild type $(100 \%)$ mice. The differences in contractile parameters and $\mathrm{Ca}^{2+}$ kinetics in transgenic cardiomyocytes and the depressed left ventricular systolic function in transgenic mice were abolished upon isoproterenol stimulation. These findings indicate that a fraction of the $\mathrm{Ca}^{2+}$-ATPases in native SR is not under regulation by phospholamban. Expression of additional phospholamban molecules results in: $(a)$ inhibition of $\mathrm{SR} \mathrm{Ca}^{2+}$ transport; (b) decreases in systolic $\mathrm{Ca}^{2+}$ levels and contractile parameters in ventricular myocytes; and (c) depression of basal left ventricular systolic function in vivo. $(J$. Clin. Invest. 1996. 97:533-539.) Key words: transgenic •
\end{abstract}

Address correspondence to Dr. Evangelia G. Kranias, Department of Pharmacology and Cell Biophysics, 231 Bethesda Ave., University of Cincinnati, OH 45267-0575. Phone: 513-558-2327; FAX: 513-5581169.

Received for publication 19 July 1995 and accepted in revised form 30 October 1995.

J. Clin. Invest.

(C) The American Society for Clinical Investigation, Inc.

0021-9738/96/01/533/07 \$2.00

Volume 97, Number 2, January 1996, 533-539 phospholamban • overexpression • cardiomyocyte • left ventricular function

\section{Introduction}

Sarcoplasmic reticulum (SR $)^{1}$ is an internal membrane system, which plays a critical role in the regulation of cytosolic $\mathrm{Ca}^{2+}$ concentrations and thus, excitation-contraction coupling in muscle. Contraction is mediated through the release of $\mathrm{Ca}^{2+}$ from the SR, while relaxation involves the active re-uptake of $\mathrm{Ca}^{2+}$ into the SR lumen by a $\mathrm{Ca}^{2+}$-ATPase. In cardiac muscle, the $\mathrm{SR} \mathrm{Ca}^{2+}$-ATPase activity is under reversible regulation by phospholamban. Dephosphorylated phospholamban inhibits the affinity of the SR $\mathrm{Ca}^{2+}$-ATPase for $\mathrm{Ca}^{2+}$ and phosphorylation relieves the inhibitory effects $(1,2)$. In vitro studies have shown that phospholamban can be phosphorylated at distinct sites by cAMP-dependent, $\mathrm{Ca}^{2+}$-calmodulin and $\mathrm{Ca}^{2+}$-phospholipid-dependent protein kinases (3-5). Each phosphorylation is associated with stimulation of SR $\mathrm{Ca}^{2+}$-transport (6). The stimulatory effects of phospholamban phosphorylation can be reversed by a SR-associated protein phosphatase activity (7). Phospholamban has also been shown to be phosphorylated in vivo and the resulting stimulatory effects on SR $\mathrm{Ca}^{2+}$ transport have been suggested to be partially responsible for the myocardial responses to $\beta$-agonists (8-10).

Recently, the role of phospholamban in the regulation of basal myocardial contractility has been elucidated through the generation of a phospholamban-deficient mouse model (11). Ablation of phospholamban was associated with significant increases in intraventricular pressure and in the rates of contraction and relaxation, assessed in isolated work-performing heart preparations (11). The elevated contractile parameters in phospholamban-deficient hearts could not be further stimulated with isoproterenol (11). These findings demonstrated that phospholamban is a repressor of left ventricular basal contractile parameters, and alterations in the levels of phospholamban would be expected to result in alterations in myocardial contractility. The critical determinant in these regulatory processes appears to be the stoichiometric ratio of phospholamban to the SR $\mathrm{Ca}^{2+}$-ATPase, which modulates the affinity of the SR $\mathrm{Ca}^{2+}$ pump for $\mathrm{Ca}^{2+}$. However, the molar stoichiometry of phospholamban/ $\mathrm{Ca}^{2+}$-ATPase in native SR is not presently known. Ratios of 1:1 (12), 2:1 (13), and 0.2:1 (14) have been reported for phospholamban/ $\mathrm{Ca}^{2+}$-ATPase, reflecting the difficulties in assessing the levels of these two proteins in SR membranes. Thus, it is not presently clear whether all the $\mathrm{Ca}^{2+}$ pumps in cardiac SR membranes are under reversible

1. Abbreviations used in this paper: $\alpha$-MHCp, $\alpha$-myosin heavy chain gene promoter; AT, acceleration time; EDD, end diastolic dimension; ESD, end systolic dimension; ET, ejection times; FS, fractional shortening; SR, sarcoplasmic reticulum; SV40, simian virus 40. 
regulation by phospholamban or whether there exists a fraction of "spare," nonregulated $\mathrm{Ca}^{2+}$-pumps. The present study was designed to critically examine the stoichiometry of phospholamban in vivo by overexpressing the protein in cardiac muscle.

Cardiac specific overexpression of the phospholamban gene in the mouse was achieved using the mouse $\alpha$-myosin heavy chain $(\alpha-\mathrm{MHCp})$ gene promoter. This promoter drives high levels of hormonally and developmentally regulated gene expression and has been successfully used in overexpression studies of adrenergic receptors in murine hearts $(15,16)$. Our hypothesis was that a fraction of the $\mathrm{SR} \mathrm{Ca}^{2+}$ pumps is not under regulation by phospholamban in native SR, and overexpression of phospholamban would be associated with inhibition of these pumps, resulting in decreased contractile parameters due to altered $\mathrm{Ca}^{2+}$ kinetics. To test this hypothesis, hearts from transgenic mice, which overexpressed phospholamban, and wild type littermate mice were analyzed in parallel at the molecular, biochemical and physiological levels. Our findings indicate that phospholamban overexpression was associated with alterations in the affinity of SR $\mathrm{Ca}^{2+}$ transport for $\mathrm{Ca}^{2+}$, which resulted in alterations in systolic free $\mathrm{Ca}^{2+}$ levels, cardiomyocyte mechanics and depressed left ventricular systolic function.

\section{Methods}

Generation and identification of transgenic mice by pronuclear injection. A 603-bp fragment encompassing $13 \mathrm{bp}$ of the 5' untranslated region, the entire phospholamban coding region and $434 \mathrm{bp}$ of the $3^{\prime}$ untranslated region of the mouse phospholamban cDNA, including one of the endogenous polyadenylation signals (nucleotides -13 to 590 in reference 17), was generated using standard PCR mediated methodology (flanked with SalI and NotI sites). This fragment was ligated, along with a 250-bp NotI-HindIII fragment containing the simian virus 40 (SV40) polyadenylation signal, into the SalI-HindIII sites of the $5.5-\mathrm{kb}$ mouse $\alpha$-MHC promoter, contained in pIBI 31 (18). The resulting construct contained the $\alpha$-MHC promoter, the entire phospholamban coding region as defined above, and the SV40 polyadenylation signal sequence (see Fig. $1 A$ ). The entire 6.4-kb KpnI-HindIII fragment was purified from plasmid sequences and used for microinjection of fertilized mouse eggs, which were subsequently implanted into pseudopregnant foster mothers (19).

Transgenic mice carrying the $\alpha$-MHC driven phospholamban transgene were identified using PCR and Southern analyses of genomic DNA isolated from tail biopsies $(20,21)$. The copy number of the transgene was determined relative to the endogenous phospholamban gene (copy number $=2$ ), using a PhosphorImager and the ImageQuant analysis system (Molecular Dynamics, Sunnyvale, CA). Mice positive for the transgene were bred and transgene expression was determined using Northern analysis $(17,22)$.

Quantitative immunoblotting of cardiac homogenates and microsomes enriched in SR membranes was carried out as previously described (23). Phospholamban was detected using IgG fractionated immune serum isolated from rabbits that had been injected with a peptide corresponding to amino acids $1-25$ of phospholamban (23). The IgG fractionated immune serum was used at a dilution of 1:25. The SR $\mathrm{Ca}^{2+}$-ATPase was detected using a 1:500 concentration of an $\mathrm{IgG}$ fraction of polyclonal antiserum (2). Briefly, hearts were homogenized at $4^{\circ} \mathrm{C}$ in buffer A composed of imidazole $(10 \mathrm{mM}, \mathrm{pH} 7.0)$, sucrose $(300 \mathrm{mM})$, dithiothreitol $(1 \mathrm{mM})$, sodium metabisulphite $(1 \mathrm{mM})$, and phenylmethylsulfonyl fluoride $(0.3 \mathrm{mM})$. Isolation of microsomal fractions enriched in SR membranes was achieved by differential centrifugation of the cardiac homogenate. Homogenates were centrifuged at $8,000 \mathrm{~g}(20 \mathrm{~min})$ and the pellets rehomogenized in buffer $\mathrm{A}$ and centrifuged as above. The supernatants from the two spins were combined, $4 \mathrm{M} \mathrm{NaCl}$ was added to a final concentration of $0.6 \mathrm{M}$, and centrifuged at $100,000 \mathrm{~g}(60 \mathrm{~min})$. The resulting pellet was washed in buffer A and recentrifuged at 100,000 $\mathrm{g}(60 \mathrm{~min})$. The final pellet was resuspended in buffer $\mathrm{A}$ and stored at $-80^{\circ} \mathrm{C}$. Protein concentration was determined by the Bio-Rad method using either bovine serum albumin or IgG as a standard. The yield of protein in the final pellet was similar between wild type and transgenic hearts. A ${ }^{35}$ S-labeled secondary anti rabbit antibody $\left(2 \times 10^{5} \mathrm{cpm} / \mathrm{ml}\right.$; Amersham Corp., Arlington Heights, IL) was used to visualize the phospholamban and SR $\mathrm{Ca}^{2+}$-ATPase proteins, which were quantitated using a PhosphorImager and the ImageQuant software.

Ventricular cardiomyocyte contractile parameters and calcium transients. Calcium tolerant mouse left ventricular myocytes from 12-13-wk-old mice were obtained as previously described (24) except for the following modifications: isolated hearts were perfused with low $\mathrm{Ca}^{2+}(25 \mu \mathrm{M})$ Joklik medium containing $75 \mathrm{U} / \mathrm{ml}$ each of Worthington collagenase type I and type II (Worthington Biochemical Corp., Freehold, NJ). The enzyme perfusion was not followed by a washout, but instead the heart was immediately removed from the perfusion apparatus and minced in low $\mathrm{Ca}^{2+}$ Joklik medium. For contractile measurements, mycoyte length and width were determined from videotaped images and calibrated with a micrometer.

For measurement of intracellular free $\mathrm{Ca}^{2+}$ transients, half of the cells from each heart were loaded with $14 \mu \mathrm{M}$ Fura- $2 \mathrm{AM}$ at $37^{\circ} \mathrm{C}$ for $30 \mathrm{~min}$ in low $\mathrm{Ca}^{2+}$ Joklik medium. After loading, cells were washed and resuspended in oxygenated physiologic buffer consisting of $\mathrm{NaCl}$ (132 mM), $\mathrm{KCl}(4.8 \mathrm{mM}), \mathrm{MgCl}_{2}(1.2 \mathrm{mM})$, glucose (5 mM), $\mathrm{CaCl}_{2}$ $(1.8 \mathrm{mM})$, and Hepes (10 mM, pH 7.2). Measurement of field-stimulated myocyte contraction was performed as previously described (25) except that the studies were performed at room temperature $\left(21^{\circ} \mathrm{C}\right)$. This lower temperature allowed the myocytes to be stable for up to $2 \mathrm{~h}$ with constant pacing and this time factor was important for the isoproterenol dose-response studies. Furthermore, the percent shortening and the rate indices are comparable between the wild type controls reported here and those in our previous study. The mechanical results are reported for cells which were not loaded with Fura-2 $\mathrm{AM}$, as the $\mathrm{Ca}^{2+}$ indicator dye was found to attenuate by $20-25 \%$ myocyte contractility (unpublished observations).

Intracellular-free $\mathrm{Ca}^{2+}$ was monitored as the ratio of $340-380 \mathrm{~nm}$ fluorescence of Fura-2 (emission wavelength $500 \mathrm{~nm}$ ) using a photo scan dual beam spectrofluoro-photometer (Photon Tech. Inc., Santa Clara, CA) coupled via fiber optic cable to an Olympus IMT-2 UV fluorescent microscope with UV transparent optics. $\mathrm{Ca}^{2+}$ data are reported as the $340 / 380 \mathrm{~nm}$ ratio, as prior studies have noted that high mitochondrial fluorescence can adversely affect calculated $\mathrm{Ca}^{2+}$ concentrations in Fura-2-loaded myocytes (25).

In some animals, the myocyte contractile and $\mathrm{Ca}^{2+}$ responses to isoproterenol were measured by sequential perfusion of $0,1,10,30$, and $100 \mathrm{nM}$ concentrations using a Harvard pump piggybacked into the perfusion apparatus. All data were collected for $40 \mathrm{~s}$ per isoproterenol dose and an additional 2 min was permitted for equilibration between dosage adjustments. With isoproterenol concentrations of $200 \mathrm{nM}$ or greater there was an unacceptable incidence of spontaneous myocyte contraction and hyper-contracture.

In vivo echocardiographic measurement of cardiac function. Echocardiographic studies were performed in 12-13-wk-old mice weighing $28 \pm 5.1 \mathrm{~g}$. The first group of mice (eight transgenic and seven wild types) were anesthetized with $0.1 \mathrm{ml} / \mathrm{mg}$ of a mixture of $65 \%$ ketamine, $22 \%$ acepromazine, and $13 \%$ xylazine (at a concentration of $10 \mathrm{mg} / \mathrm{ml}$ ). In order to obtain more physiological heart rates, a second group of mice (seven transgenic and seven wild types) were lightly anesthetized with $2.5 \%$ avertin $(0.01 \mathrm{ml} / \mathrm{g})$. In all instances, animals were allowed to breathe spontaneously.

Two-dimensional targeted M-mode and color flow-directed pulsed wave Doppler studies were performed with a $9 \mathrm{MHz}$ imaging and a 5-7.5 $\mathrm{MHz}$ Doppler transducer (Interspec-ATL Apogee X-200) as previously described (26). Studies were performed at baseline and after 
the administration of $2.0 \mu \mathrm{g} / \mathrm{g}$ i.p. isoproterenol. M mode measurements of end diastolic dimension (EDD) and end systolic dimension (ESD) were made from original tracings, and Doppler measurements of peak (Ao) and integrated $\left(\mathrm{Ao}_{\mathrm{vti}}\right)$ aortic velocities, acceleration (AT), and ejection times (ET), were made from $1 / 2$ inch videotapes. Calculated variables included: left ventricular (LV) fractional shortening (FS = EDD-ESD/EDD), normalized mean velocity of circumferential fiber shortening $\left(\mathrm{V}_{\mathrm{cfc}}=\mathrm{FS} / \mathrm{ET}\right)$ and mean aortic acceleration $(\mathrm{Acc}=\mathrm{Ao} / \mathrm{AT})$. The percent change from baseline of these variables with isoproterenol administration was computed as: $100 \times$ (isoproterenol value - baseline value)/baseline value.

Calcium uptake assays. Hearts from 12-13-wk-old mice were excised, rinsed in ice cold phosphate buffered saline, frozen in liquid nitrogen, and stored at $-80^{\circ} \mathrm{C}$. The frozen hearts were homogenized at $4^{\circ} \mathrm{C}$ and $\mathrm{Ca}^{2+}$ uptake measurements were performed as previously described (11).

Statistical analysis. Data are presented as mean \pm SE. The number $(n)$ of mice used is indicated. For the myocyte experiments, multiple cells (at least three myocytes/heart) from individual hearts were treated as replicate determinations and each animal was considered as a single $n$. Statistical analysis was performed by $t$ test between wild type and transgenic mice.

\section{Results}

Generation of mice overexpressing phospholamban in the heart. The mouse $\alpha$-MHC gene promoter was used to direct

(A)

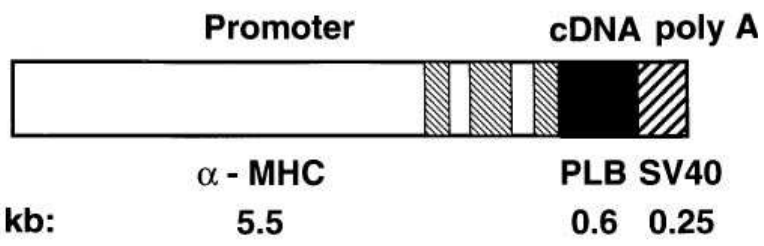

(B)

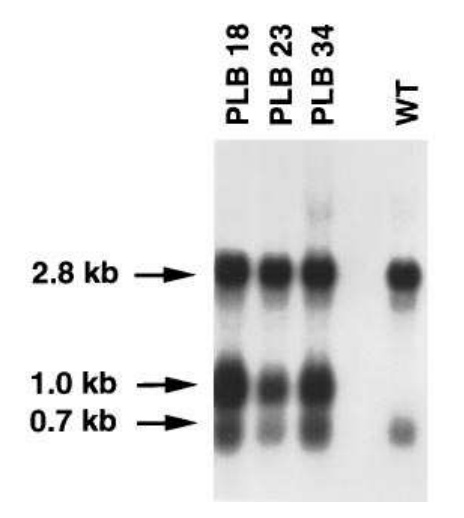

Figure 1. (A) Schematic representation of the $\alpha$-MHCp-phospholamban-SV-40 fusion gene used for the generation of transgenic mice overexpressing phospholamban. The hatched regions in the $\alpha$-MHCp represent $\alpha$-MHC exons 1, 2, and part of 3. (B) Northern blot analysis of total RNA $(10 \mu \mathrm{g})$ from three transgenic lines (PLB 18, PLB 23, and $P L B 34)$ and wild type (WT) littermates. The RNA was denatured, electrophoresed on a $1.2 \%$ denaturing agarose gel containing 2.2 M formaldehyde, blotted onto GeneScreen Plus membranes (Dupont NEN, Boston, MA), and probed with a ${ }^{32} \mathrm{P}$-labeled phospholamban cDNA fragment. The blot was washed at room temperature for 5 $\min$ in $0.5 \times \mathrm{SSC}$ and $0.5 \%$ SDS, followed by washing at $60^{\circ} \mathrm{C}$ for $1 \mathrm{~h}$ in $0.5 \times$ SSC, and $0.5 \%$ SDS. The endogenous mouse phospholamban transcripts migrate at 2.8 and $0.7 \mathrm{~kb}$, while the transgenic mRNA migrates at $1.0 \mathrm{~kb}$.

expression of mouse phospholamban cDNA in the heart. Five transgenic lines were established and Southern blot analysis of tail DNA indicated that the copy number of the transgene ranged between two to nine copies per line. Transgene expression was assessed by Northern blot analysis of total cardiac RNA from the five germ lines, using phospholamban cDNA as a probe. The two major endogenous phospholamban transcripts, migrating at 0.7 and $2.8 \mathrm{~kb}$ were detected in all lines and three out of the five transgenic lines demonstrated intense expression of an additional transcript migrating at $1.0 \mathrm{~kb}$ (Fig. $1 \mathrm{~B}$ ). The transgenic origin of the $1.0-\mathrm{kb}$ phospholamban transcript was confirmed by Northern blot analysis, using a synthetic oligonucleotide (60 bases) probe containing 30 bases of the $5^{\prime}$ untranslated region from the $\alpha$-MHC promoter and 30 bases of the $5^{\prime}$ region from the phospholamban cDNA. Two of the transgenic lines (copy number: 2 and 3, respectively) reproduced efficiently and were propagated for further characterization.

To quantitate the levels of phospholamban protein expression, cardiac homogenates from transgenic, and wild type mice were processed in parallel for Western blot analyses. Quantitative immunoblotting revealed a twofold increase in the levels of phospholamban in transgenic hearts compared to wild type hearts (Fig. 2 B). To determine whether the overexpressed phospholamban was incorporated in SR, microsomal preparations enriched in SR membranes were isolated from transgenic and wild type hearts and studied in parallel with their respective homogenates. The levels of phospholamban and the relative ratio of phospholamban/SR $\mathrm{Ca}^{2+}$ pump were twofold higher (at each protein concentration loaded) in both the homogenates (Fig. $2 \mathrm{C}$ ) and the microsomes from transgenic hearts compared to those present in wild type hearts, indicating that the overexpressed phospholamban was incorporated in the SR membranes.

(A)

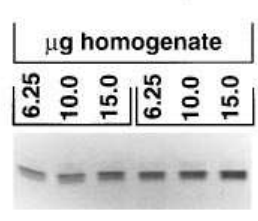

(B)

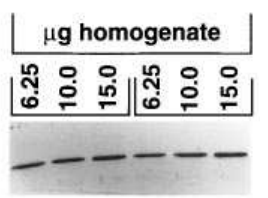

(C)

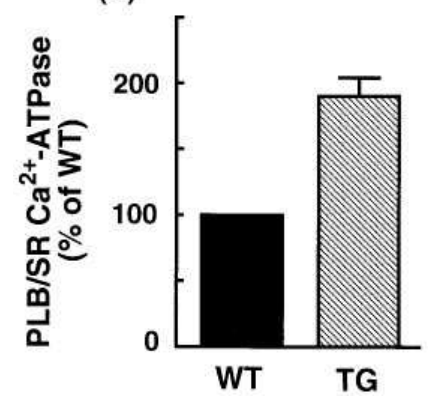

Figure 2. Western blot of cardiac homogenates $(6.25,10$, and $15 \mu \mathrm{g}$ ) from transgenic mice and wild type (WT) littermates. Total cardiac homogenate protein was electrophoresed on a $10-20 \%$ gradient sodium dodecyl sulfate polyacrylamide gel, electroblotted onto a nitrocellulose membrane (Schleicher \& Schuell, Keene, $\mathrm{NH})$ and $(A)$ probed with a phospholamban polyclonal antibody followed by a ${ }^{35}$ S-labeled anti-rabbit secondary antibody. $(B)$ probed with a SR $\mathrm{Ca}^{2+}$-ATPase polyclonal antibody followed by a ${ }^{35}$ S-labeled anti-rabbit secondary antibody. The phospholamban and SR $\mathrm{Ca}^{2+}$-ATPase protein levels were quantitated as described in Methods. $(C)$ levels of phospholamban protein in transgenic mice $(n=3)$ compared to wild type littermates expressed as the relative ratio of PLB/SR $\mathrm{Ca}^{2+}$-ATPase, at each protein concentration loaded (6.25, 10 , and $15 \mu \mathrm{g}$ ). Values represent the mean $\pm \mathrm{SE}$. 


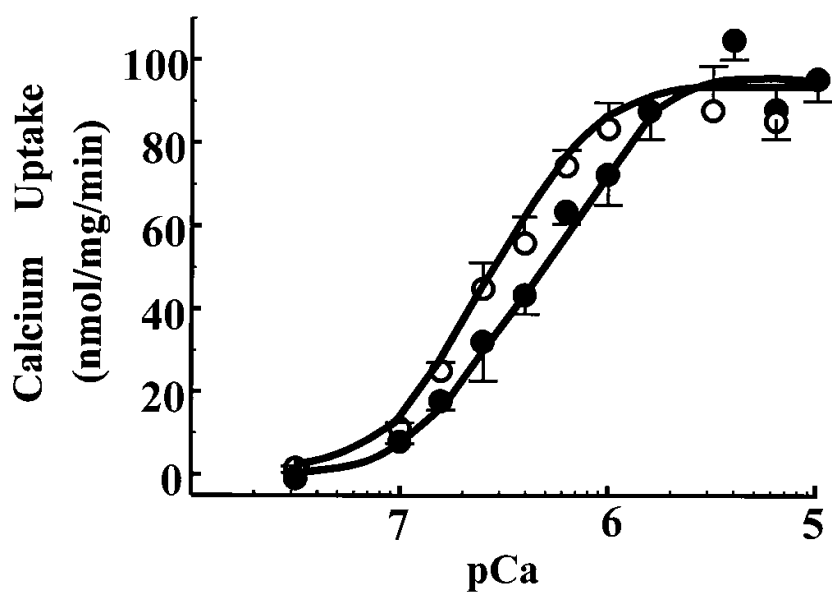

Figure 3. The effect of phospholamban overexpression on the affinity of the SR $\mathrm{Ca}^{2+}$-ATPase for $\mathrm{Ca}^{2+}$. The initial rates of $\mathrm{Ca}^{2+}$ uptake by $\mathrm{SR}$ vesicles in mouse cardiac homogenates from transgenic $(\bullet, \mathrm{TG}, n=$ $4)$ and wild type $(\mathrm{O}, \mathrm{WT}, n=3)$ mice were assayed over a wide range of $\mathrm{Ca}^{2+}$ concentrations. Data represent the mean $\pm \mathrm{SE}$.

Sarcoplasmic reticulum calcium uptake rates. The effects of phospholamban overexpression on the initial rates of ATP dependent, oxalate facilitated SR $\mathrm{Ca}^{2+}$ uptake were assessed using cardiac homogenates. The conditions of the assay, which restrict the uptake of $\mathrm{Ca}^{2+}$ into the $\mathrm{SR}$, have previously been defined (27). $\mathrm{Ca}^{2+}$ uptake rates by the homogenates of the transgenic mouse hearts were significantly lower than those by the wild type hearts. However, there was no significant change in the maximum velocity of $\mathrm{Ca}^{2+}$ uptake $\left(V_{\text {max }}\right)$ in the transgenic hearts compared to wild type hearts (Fig. 3). Analysis of these data indicates that the $\mathrm{EC}_{50}$ of the SR $\mathrm{Ca}^{2+}$-ATPase for $\mathrm{Ca}^{2+}$ was significantly higher in the transgenic hearts $(0.48 \pm 0.04, n=4)$ compared to wild type hearts $(0.27 \pm 0.01$, $n=3)$.

Contractile parameters in isolated cardiomyocytes. To determine whether the decreased affinity of the SR $\mathrm{Ca}^{2+}$ pump for $\mathrm{Ca}^{2+}$ upon phospholamban overexpression was associated with alterations in contractile parameters, left ventricular myocytes from transgenic and wild type hearts were isolated, paced at $0.25 \mathrm{~Hz}$ and their mechanical properties were examined. Table I shows the mechanical properties of myocytes from 12-13wk-old transgenic hearts and their littermate wild type hearts. There were no alterations in either the cell length or cell width of isolated cardiomyocytes upon phospholamban overexpression in the heart. However, the contractile parameters of transgenic cardiomyocytes were significantly depressed in comparison to those in wild type littermates. The extent of cardiomyocyte shortening and the rates of shortening $(+\mathrm{dL} /$ $\mathrm{dt})$ and relengthening $(-\mathrm{dL} / \mathrm{dt})$ were each depressed by 37,36 , and $45 \%$, respectively, in the transgenic cardiomyocytes compared to wild type cardiomyocytes.

Calcium transients in isolated cardiomyocytes. The observed changes in contractile parameters suggested alterations in the $\mathrm{Ca}^{2+}$ kinetics of transgenic myocytes. Thus, cardiomyocytes were loaded with Fura-2 AM and the phasic $\mathrm{Ca}^{2+}$ signals during pacing were examined (Fig. 4). In transgenic cardiomyocytes, the amplitude of the $\mathrm{Ca}^{2+}$ signal during systole was depressed, and the time for decay of this signal, which represents at least in part the uptake of $\mathrm{Ca}^{2+}$ by $\mathrm{SR}$, was prolonged com-
Table I. Contractile Parameters and $\mathrm{Ca}^{2+}$ Transients in Isolated Unloaded and Fura-2-loaded Left Ventricular Cardiomyocytes from Transgenic Mice and Wild Type Littermates

\begin{tabular}{lccl}
\hline & Wild type & Transgenic & $P$ value \\
\hline Contractile parameters & & & \\
$\quad$ Percentage Shortening & $9.2 \pm 0.7$ & $5.8 \pm 0.7$ & 0.004 \\
$+\mathrm{dL} / \mathrm{dt}, \mu \mathrm{m} / \mathrm{s}$ & $167 \pm 16$ & $106 \pm 15$ & 0.014 \\
$\quad-\mathrm{dL} / \mathrm{dt}, \mu \mathrm{m} / \mathrm{s}$ & $125 \pm 14$ & $69 \pm 12$ & 0.007 \\
Calcium kinetics & & & \\
$\quad$ Amplitude, $340 / 380 \mathrm{~nm}$ & $0.71 \pm 0.06$ & $0.59 \pm 0.04$ & 0.08 \\
$\mathrm{~T}_{80 \%}, \mathrm{~s}$ & $0.59 \pm 0.06$ & $0.77 \pm 0.04$ & 0.017 \\
& & &
\end{tabular}

Nine wild type hearts were used for the contractile parameters and $\mathrm{Ca}^{2+}$ kinetics, while 11 and 10 transgenic hearts were used for the contractile parameters and $\mathrm{Ca}^{2+}$ kinetics, respectively. Values represent mean $\pm \mathrm{SE}$ with $P$ value assessed by $t$ test between wild type and transgenic mice.

$+\mathrm{dL} / \mathrm{dt}$, rate of cell shortening.

$-\mathrm{dL} / \mathrm{dt}$, rate of cell relengthening.

$\mathrm{T}_{80 \%}$, time for $80 \%$ decay of the $\mathrm{Ca}^{2+}$ signal.

pared to wild type cardiomyocytes. Analyses of these data, obtained in cardiomyocytes from multiple transgenic and wild type hearts, indicated that the amplitude of the systolic $\mathrm{Ca}^{2+}$ transient was lower and the time for $80 \%\left(\mathrm{~T}_{80 \%}\right)$ decay of this signal was significantly prolonged in transgenic cardiomyocytes compared to wild type myocytes (Table I). However, the level of diastolic free $\mathrm{Ca}^{2+}$ concentration was similar between transgenic and wild type cardiomyocytes, suggesting that although the rate of SR $\mathrm{Ca}^{2+}$ uptake was slower upon phospholamban overexpression, the $\mathrm{Ca}^{2+}$ capacity of the SR was not altered.

Isoproterenol effects on contractile parameters and $\mathrm{Ca}^{2+}$ transients in cardiomyocytes. Phospholamban has been postulated to play a major role in the cardiac responses to $\beta$-agonist stimulation (8-10). Thus, cardiomyocytes from transgenic and wild type mice were subjected to incremental dosage of isoproterenol and their contractile and $\mathrm{Ca}^{2+}$-signaling parameters were measured. It is interesting to note that although the basal rates of shortening $(+\mathrm{dL} / \mathrm{dt})$ and relengthening $(-\mathrm{dL} / \mathrm{dt})$ in transgenic cardiomyocytes were significantly depressed, these parameters could be stimulated by isoproterenol. The maximally stimulated contractile parameters, obtained at $0.1 \mu \mathrm{M}$

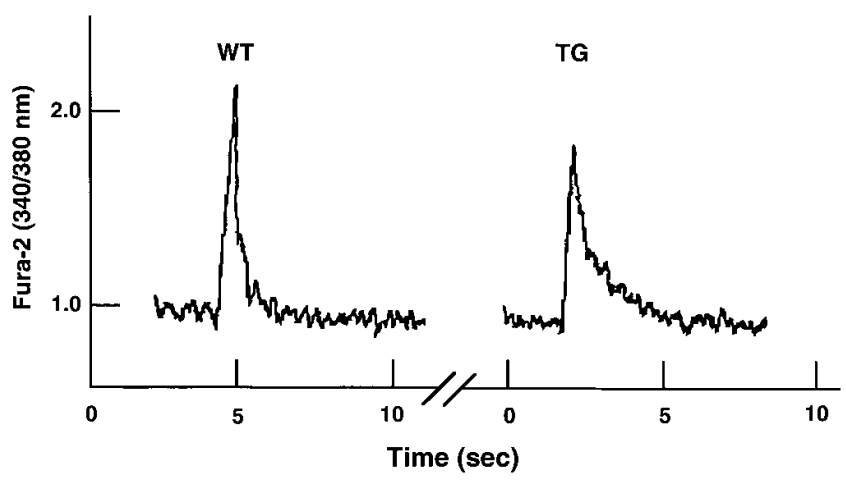

Figure 4. Representative recording of the $\mathrm{Ca}^{2+}$ transients in Fura-2loaded cardiomyocytes from wild type $(W T)$ and transgenic $(T G)$ mice paced at $0.25 \mathrm{~Hz}$. 
(A)

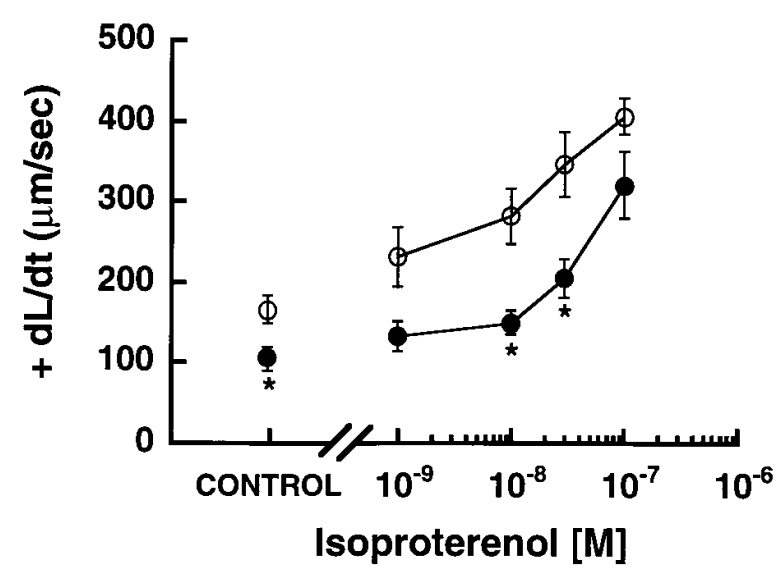

(B)

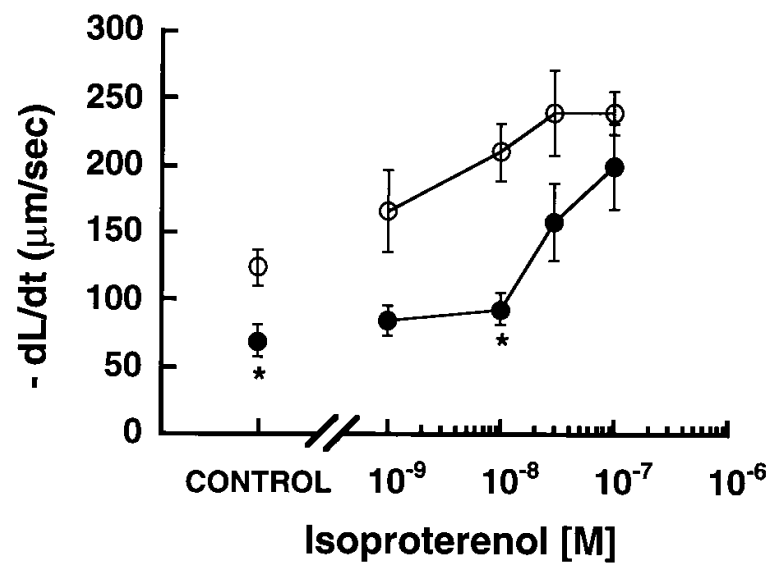

Figure 5. Effects of incremental doses of isoproterenol on $(A)$ rate of shortening $(+d L / d t)$ and $(B)$ rate of relengthening $(-d L / d t)$ in unloaded cardiomyocytes from transgenic $(\mathbf{0}, \mathrm{TG})$ and wild type $(\bigcirc$, WT) mice, paced at $0.25 \mathrm{~Hz}$. Values represent the mean \pm SE. Control values were obtained using $9 \mathrm{WT}$ and $11 \mathrm{TG}$ hearts. Isoproterenol responses were obtained using $4 \mathrm{WT}$ and 3 TG hearts. ${ }^{*} P<0.05$.

isoproterenol, were similar between transgenic and wild type cardiomyocytes (Fig. 5, $A$ and $B$ ). Similarly, under maximal isoproterenol stimulation, the amplitude of the systolic $\mathrm{Ca}^{2+}$ transient, which represents the total free systolic $\mathrm{Ca}^{2+}$, and the rate of decay of this signal were similar between transgenic and wild type cardiomyocytes (Fig. 6, $A$ and $B$ ).

In vivo assessment of left ventricular systolic function in mice. In order to determine whether the depressed mechanical properties and altered $\mathrm{Ca}^{2+}$ kinetics observed in isolated cardiomyocytes from transgenic mice exhibited similar depression in left ventricular systolic function in vivo, $M$ mode and Doppler echocardiography was performed in transgenic and wild type mice. Table II shows the parameters of left ventricular function, from 12-13-wk-old transgenic and wild type mice, studied in parallel. The ESD (115\%) was significantly higher while the FS $(21 \%)$ and $V_{\text {cfc }}(33 \%)$ were significantly depressed in transgenic mice as compared to wild type (100\%) mice. There was also a trend $(P=0.09)$ towards lower mean Acc in transgenic mice compared to wild type mice. However,

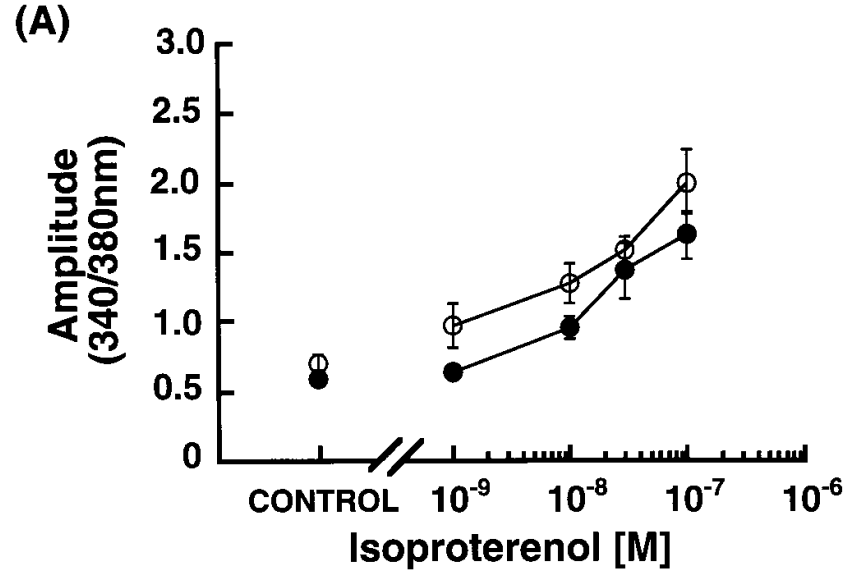

(B)

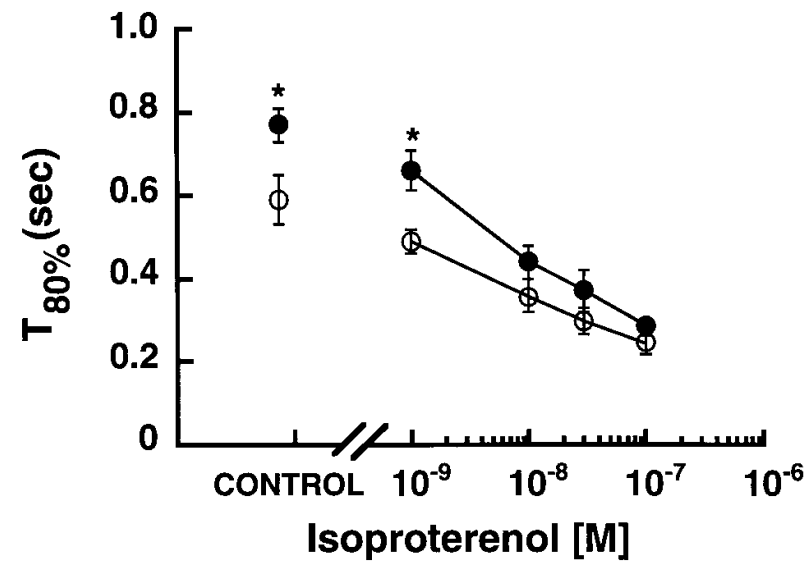

Figure 6. Effects of incremental doses of isoproterenol on $(A)$ amplitude of the $\mathrm{Ca}^{2+}$ signal and $(B)$ time for $80 \%$ decline of this signal $\left(\mathrm{T}_{80 \%}\right)$ in Fura-2-loaded cardiomyocytes from transgenic $(\bullet, \mathrm{TG})$ and wild type $(\bigcirc, W T)$ mice, paced at $0.25 \mathrm{~Hz}$. Values represent the mean \pm SE. Control values were obtained using $9 \mathrm{WT}$ and $10 \mathrm{TG}$ hearts. Isoproterenol responses were obtained using $4 \mathrm{WT}$ and $3 \mathrm{TG}$ hearts. $* P<0.05$

the heart rate, EDD, $\mathrm{Ao}_{\mathrm{VTI}}$, Ao, and the E/A ratio were similar between the two groups.

To determine the effects of isoproterenol on transgenic left ventricular systolic function, mice were subjected to intraperitoneal administration of this $\beta$-adrenergic agonist and $\mathrm{M}$ mode and Doppler measurements were repeated (Table II). Isoproterenol caused significant increases in heart rate, left ventricular FS, $\mathrm{V}_{\mathrm{cfc}}$, Ao, and Acc in both transgenic and wild type mice. The percent increase in FS, $\mathrm{V}_{\mathrm{cfc}}$, and Acc were significantly greater in transgenic than in wild type mice, consistent with the increased levels of phospholamban in the overexpression hearts. However, the relative increases in heart rate were similar between transgenic and wild type mice, suggesting that phospholamban has little influence on sinus node activity.

\section{Discussion}

The present study demonstrates that phospholamban overexpression in the heart is associated with decreases in the affinity 
Table II. M Mode Echocardiographic and Doppler

Measurements of Basal and Isoproterenol Stimulated, Left Ventricular Systolic Function in Transgenic and Wild Type Mice

\begin{tabular}{|c|c|c|c|c|}
\hline & \multicolumn{2}{|c|}{ Wild type } & \multicolumn{2}{|c|}{ Transgenic } \\
\hline & Pre & Post & Pre & Post \\
\hline EDD (mm) & $3.8 \pm 0.1$ & $3.0 \pm 0.1 *$ & $3.6 \pm 0.1$ & $3.3 \pm 0.1^{*}$ \\
\hline $\operatorname{ESD}(\mathrm{mm})$ & $2.0 \pm 0.1$ & $1.02 \pm 0.1 *$ & $2.3 \pm 0.1$ & $1.2 \pm 0.01 *$ \\
\hline FS (\%) & $44 \pm 1.6$ & $66 \pm 2.0^{*}$ & $35 \pm 2.0^{\ddagger}$ & $64 \pm 1.0 *$ \\
\hline $\mathrm{V}_{\mathrm{cfc}}(\operatorname{circ}-1)$ & $6.2 \pm 0.5$ & $11.5 \pm 0.6^{*}$ & $4.2 \pm 0.3^{\ddagger}$ & $10.4 \pm 0.5^{*}$ \\
\hline Ao $(\mathrm{cm} / \mathrm{sec})$ & $71.2 \pm 3.4$ & $88.6 \pm 4.7 *$ & $66.5 \pm 2.2$ & $92.5 \pm 3.0^{*}$ \\
\hline $\mathrm{Ao}_{\mathrm{VTI}}(\mathrm{cm})$ & $3.1 \pm 0.2$ & $3.0 \pm 0.1$ & $3.3 \pm 0.2$ & $3.6 \pm 0.2$ \\
\hline HR (bpm) & $292 \pm 23$ & $452 \pm 18^{*}$ & $280 \pm 25$ & $431 \pm 25^{*}$ \\
\hline $\operatorname{Acc}(\mathrm{m} / \sec 2)$ & $37 \pm 4.0$ & $60 \pm 6.0^{*}$ & $29 \pm 2.0$ & $66 \pm 4.0 *$ \\
\hline
\end{tabular}

14 wild type mice and 15 transgenic mice were used for $\mathrm{M}$ mode echocardiographic and Doppler measurements of left ventricular function at baseline (Pre) and after isoproterenol stimulation (Post). Values represent mean \pm SE with $\mathrm{P}$ value assessed by $t$ test between wild type and transgenic mice.

$* \mathrm{P}<0.05$ vs. Pre Iso.

${ }^{\ddagger} \mathrm{P}<0.05$ vs. wild type.

of the SR $\mathrm{Ca}^{2+}$-ATPase for $\mathrm{Ca}^{2+}$, which results in altered systolic $\mathrm{Ca}^{2+}$ and contractile parameters of cardiomyocytes and depressed left ventricular systolic function in vivo. This is the first evidence indicating that a fraction of the $\mathrm{Ca}^{2+}$ pumps in cardiac SR is not under regulation by phospholamban, such that expression of additional phospholamban molecules results in further modulation of this enzymatic activity and overall myocardial contractility.

The ability to assess $\mathrm{Ca}^{2+}$ kinetics and contractile parameters in isolated ventricular cardiomyocytes enabled us to link biochemical alterations at the SR level with physiological alterations at the cellular level in transgenic hearts. Furthermore, the isolated cardiomyocytes provided a preparation devoid of the effects of left ventricular chamber geometry, fiber orientation, and the extracellular matrix for examining the effects of phospholamban overexpression. The $\alpha$-MHC promoter, which is both developmentally and hormonally regulated in vivo, was used to drive overexpression of phospholamban in mouse hearts (18). Quantitative immunoblotting of cardiac homogenates and microsomes enriched in SR membranes from transgenic mice revealed a twofold increase in the phospholamban protein levels compared to wild type littermates. The relative ratio of phospholamban/SR $\mathrm{Ca}^{2+}$-ATPase was also twofold higher in the transgenic hearts compared to wild type hearts, and this was associated with a decrease in the overall $\mathrm{Ca}^{2+}$ affinity of the SR transport system. These findings together with our previous studies, in phospholamban deficient mouse hearts (11) and in rat hearts at various thyroid states (29) indicate that there is an inverse relationship between the phospholamban/ $\mathrm{Ca}^{2+}$-ATPase ratio and the affinity of the $\mathrm{Ca}^{2+}$ pump for $\mathrm{Ca}^{2+}$. Although we cannot presently exclude the possibility that the phospholamban regulatory effects on SR $\mathrm{Ca}^{2+}$ uptake in transgenic hearts may be mediated through formation of $\mathrm{Ca}^{2+}$-selective phospholamban channels, our findings on modulation of only the affinity of the transport system for $\mathrm{Ca}^{2+}$ without any effects on its maximal velocity, ar- gue that the observed regulation was mainly mediated through modulation of the SR $\mathrm{Ca}^{2+}$-ATPase activity.

The inhibitory effects of phospholamban overexpression, observed in vitro, reflected physiological alterations assessed in ventricular cardiomyocytes. Both the rate of the $\mathrm{Ca}^{2+}$ signal decline and the rate of relaxation were significantly prolonged in isolated cardiomyocytes from transgenic hearts compared to wild type hearts. Consistent with the increased inhibition of SR $\mathrm{Ca}^{2+}$ uptake rates and decreased levels of $\mathrm{SR} \mathrm{Ca}^{2+}$ available for contraction, the transgenic cardiomyocytes exhibited decreases in the amplitude of the $\mathrm{Ca}^{2+}$ signal and their extent of shortening compared to wild type cardiomyocytes. These inhibitory effects of phospholamban overexpression, observed in ventricular cardiomyocytes, are expected to be of even greater magnitude in atrial cardiomyocytes. Murine atrial cardiomyocytes have a lower phospholamban/SR $\mathrm{Ca}^{2+}$-ATPase transcript abundance, which appears to be associated with enhancement of atrial basal contractile parameters, compared to ventricular cardiomyocytes (28). Thus, overexpression of phospholamban, driven by the $\alpha$-MHC promoter, is expected to result in similar levels of phospholamban in the two cardiac compartments as suggested by our in situ hybridization studies (unpublished observations), and may be associated with greater inhibition of the basal contractile parameters in atrial than ventricular tissues. Future studies, using muscle strips or cardiomyocyte preparations from atrial and ventricular tissues will provide evidence along these lines and further elucidate the physiological role of phospholamban in the two cardiac compartments.

An important question is whether depressed cardiomyocyte function by phospholamban overexpression would translate into decreased cardiac function in vivo. Thus, $\mathrm{M}$ mode and pulsed-wave Doppler echocardiography was used to noninvasively assess left ventricular function in anesthetized transgenic and age matched wild type mice (26). Basal left ventricular systolic function was significantly depressed in mice overexpressing phospholamban, consistent with findings in isolated cardiomyocytes. However, there were no changes in the diastolic filling parameters (i.e., diastolic transmitral velocities, data not shown), suggesting that either phospholamban does not modulate these parameters or that phospholamban effects are masked by the multiple determinants of the early and latetransmitral velocities (30).

Phospholamban has also been implicated as one of the key phosphoproteins in mediating the contractile responses of the heart to $\beta$-adrenergic stimulation (8-10). Thus, it was of special interest to examine the effects of isoproterenol on the depressed basal contractile parameters and $\mathrm{Ca}^{2+}$ transients in isolated left ventricular cardiomyocytes, as well as on the depressed left ventricular systolic function in transgenic mice overexpressing phospholamban, and compare them to the wild type mice. At maximal isoproterenol stimulation, the contractile parameters and $\mathrm{Ca}^{2+}$ kinetics in transgenic cardiomyocytes were similar to those in wild type cardiomyocytes. Similarly, in vivo, left ventricular systolic function, was similar in transgenic and wild type mice following isoproterenol stimulation, suggesting that the inhibitory effects of phospholamban overexpression could be overcome, presumably, due to phosphorylation of phospholamban. These findings suggest that phospholamban is an important mediator of the heart's responses to $\beta$-adrenergic agonists and are consistent with our previous studies in phospholamban deficient hearts, which exhibited 
loss of $\beta$-agonist stimulation (11). However, it remains to be determined whether phospholamban overexpression is associated with any alterations in the phosphorylation levels of troponin I (31) and phospholemma (32), the two other key regulatory phosphoproteins, which have been shown to contribute to the stimulatory effects of $\beta$-agonists.

In summary, our findings indicate that a fraction of the SR $\mathrm{Ca}^{2+}$-ATPases is not subject to regulation by phospholamban in the wild type mouse heart and overexpression of phospholamban is associated with alterations in $\mathrm{SR} \mathrm{Ca}^{2+}$ transport rates, systolic $\mathrm{Ca}^{2+}$ levels and contractile parameters. These results together with our previous studies in phospholamban deficient hearts provide strong evidence for the functional significance of phospholamban in the heart. Furthermore, the generation of an animal model with increases in the relative ratio of phospholamban/SR $\mathrm{Ca}^{2+}$-ATPase, similar to previous observations in hypertrophy (33) and heart failure (34), provides an attractive system for future studies designed to elucidate the significance of this altered stoichiometry under pathophysiological conditions. However, it remains to be determined whether compensatory mechanisms such as changes in $\mathrm{Ca}^{2+}$ cycling proteins, contractile proteins and proteins in the $\beta$-adrenergic signal transduction pathway have occurred as a result of phospholamban overexpression in the transgenic hearts.

\section{Acknowledgments}

We wish to thank Dr. J. Robbins for providing clone 20 containing the murine $\alpha$-MHC promoter. We are also grateful to J. C. Neuman in the Transgenic Core Facility of the University of Cincinnati, College of Medicine (Cincinnati, OH) for pronuclear injections. We wish to also thank Drs S. F. Khoury and Z. M. Khan for assistance with the Echocardiography studies.

This work was supported by National Institutes of Health grants HL 26057, HL 22619, HL 49267, HL 07382, and HL52318.

\section{References}

1. Hicks, M. J., M. Shigekawa, and A. M. Katz. 1979. Mechanism by which cyclic adenosine $3^{\prime}: 5^{\prime}$-monophosphate-dependent protein kinase stimulates calcium transport in cardiac sarcoplasmic reticulum. Circ. Res. 44:384-391.

2. Kim, H. W., N. A. E. Steenaart, D. G. Ferguson, and E. G. Kranias. 1990. Functional reconstitution of the cardiac sarcoplasmic reticulum $\mathrm{Ca}^{2+}$-ATPase with phospholamban in phospholipid vesicles. J. Biol. Chem. 265:1702-1709.

3. Kirchberger, M. A., M. Tada, and A. M. Katz. 1974. Adenosine $3^{\prime}: 5^{\prime}$ : monophosphate dependent protein kinase-catalyzed phosphorylation reaction and its relationship to calcium-transport in cardiac sarcoplasmic reticulum. $J$. Biol. Chem. 249:6166-6173.

4. LePeuch, C. L., J. Haiech, and J. G. Demaille. 1979. Concerted regulation of cardiac sarcoplasmic reticulum calcium transport by cyclic adenosine monophosphate-dependent and calcium-calmodulin-dependent phosphorylation. Biochemistry. 18:5150-5157.

5. Movsesian, M. A., M. Nishikawa, and R. S. Adelstein. 1984. Phosphorylation of phospholamban by calcium-activated phospholipid-dependent protein kinase. J. Biol. Chem. 259:8029-8032.

6. Davis, B. A., I. Edes, R. C. Gupta, E. F. Young, H. W. Kim, N. A. E. Steenart, G. Szymanska, and E. G. Kranias. 1990. The role of phospholamban in the regulation of calcium transport by cardiac sarcoplasmic reticulum. Mol. Cell. Biochem. 99:83-88.

7. Kranias, E. G. 1985. Regulation of $\mathrm{Ca}^{2+}$ transport by phosphoprotein phosphatase activity associated with cardiac sarcoplasmic reticulum. J. Biol. Chem. 260:11006-11010.

8. Kranias, E. G., and R. J. Solaro. 1982. Phosphorylation of troponin I and phospholamban during catecholamine stimulation of rabbit heart. Nature (Lond.). 298:182-184.

9. Lindemann, J. P., L. R. Jones, D. R. Hathaway, B. G. Henry, and A. M. Watanabe. 1983. $\beta$-Adrenergic stimulation of phospholamban phosphorylation and $\mathrm{Ca}^{2+}$-ATPase activity in guinea pig ventricles. J. Biol. Chem. 258:464-471.

10. Wegner, A. D., H. K. B. Simmerman, J. P. Lindemann, and L. R. Jones.
1989. Phospholamban phosphorylation in intact ventricles. J. Biol. Chem. 264: 11468-11474.

11. Luo, W., I. L. Grupp, J. Harrer, S. Ponniah, G. Grupp, J. J. Duffy, T. Doetschman, and E. G. Kranias. 1994. Targeted ablation of the phospholamban gene is associated with markedly enhanced myocardial contractility and loss of B-agonist stimulation. Circ. Res. 75:401-409.

12. Tada, M., M. Inui, M. Yamada, M. A. Kadoma, T. Kuzuya, H. Abe, and S. Kakiuchi. 1983. Effects of phospholamban phosphorylation catalyzed by adenosine $3^{\prime}: 5^{\prime}$-monophosphate and calmodulin-dependent protein kinases on calcium transport ATPase of cardiac sarcoplasmic reticulum. J. Mol. Cell. Cardiol. 15:335-346.

13. Colyer, J., and J. H. Wang. 1991. Dependence of cardiac sarcoplasmic reticulum calcium pump activity on the phosphorylation status of phospholamban. J. Biol. Chem. 266:17486-17493.

14. Louis, C. F., J. Turnquist, and B. Jarvis. 1987. Phospholamban stoichiometry in canine cardiac muscle sarcoplasmic reticulum. Neurosci. Res. 12:937941.

15. Milano, C. A., L. F. Allen, H. A. Rockman, P. C. Dolber, T. R. McMinn, K. R. Chien, T. D. Johnson, R. A. Bond, and R. J. Lefkowitz. 1994. Enhanced myocardial function in transgenic mice overexpressing the beta 2-adrenergic receptor. Science (Wash. DC). 264:582-586.

16. Milano, C. A., P. C. Dolber, H. A. Rockman, R. A. Bond, M. E. Venable, L. F. Allen, and R. J. Lefkowitz. 1994. Myocardial expression of a constitutively active alpha $1 \mathrm{~B}$-adrenergic receptor in transgenic mice induces cardiac hypertrophy. Proc. Natl. Acad. Sci. USA. 91:10109-10113.

17. Ganim, J., W. S. Luo, S. Ponniah, H. W. Kim, D. G. Ferguson, V. Kadambi, J. Neumann, T. Doetschman, and E. G. Kranias. 1992. Mouse phospholamban gene expression during development in vivo and in vitro. Circ. Res. 71: 1021-1030.

18. Subramaniam, A., W. K. Jones, J. Gulick, S. Wert, J. Neumann, and J. Robbins. 1991. Tissue-specific regulation of the $\alpha$-myosin heavy chain gene promoter in transgenic mice. J. Biol. Chem. 266:24613-24620.

19. Hogan, B., F. Constantini, and E. Lacey. 1986. Manipulating the Mouse Embryo. Cold Spring Harbor Laboratory, Cold Spring Harbor, New York. $332 \mathrm{pp}$.

20. Laird, P. W., A. Zijderveld, K. Linders, M. A. Rudnicki, A. Jaenisch, and A. Berns. 1991. Simplified mammalian DNA isolation procedure. Nucleic Acids Res. 19:4293-4295.

21. Walter, C. A., D. Nasr-Schirf, and V. J. Luna. 1989. Identification of transgenic mice carrying the CAT gene with PCR amplification. Biotechniques. 7:1065-1067.

22. Chomczynski, P., and N. Sacchi. 1987. Single-step method of RNA isolation by acid quanidium thiocyanate-phenol-chloroform extraction. Anal. Biochem. 162:156-159.

23. Harrer, J. M., E. Kiss, and E. G. Kranias. 1995. Application of the immunoblot technique for quantitation of protein levels in cardiac homogenates. Biotechniques. 18:995-997.

24. Dorn, II, G. W., J. Robbins, N. Ball, and R. A. Walsh. 1994. Myosin heavy chain regulation and myocyte contractile depression after LV hypertrophy in aortic-banded mice. Am. J. Physiol. 267:H400-H405.

25. Williford, D. J., V. K. Sharma, M. Korth, and S. S. Sheu. 1990. Spatial heterogeneity of intracellular $\mathrm{Ca}^{2+}$ concentration in nonbeating guinea pig ventricular myocytes. Circ. Res. 66:234-241.

26. Hoit, B. D., S. F. Khoury, E. G. Kranias, N. Ball, and R. A. Walsh. 1995. In vivo echocardiographic detection of enhanced left ventricular function in gene-targeted mice with phospholamban deficiency. Circ. Res. 77:632-637.

27. Solaro, R. J., and F. N. Briggs. 1974. Estimating the functional capabilities of sarcoplasmic reticulum in cardiac muscle. Circ. Res. 34:531-540.

28. Koss, K. L., S. Ponniah, W. K. Jones, I. L. Grupp, and E. G. Kranias. 1995. Differential phospholamban gene expression in murine cardiac compartment: molecular and physiological analyses. Circ. Res. 77:342-353.

29. Kiss, E., G. Jakab, E. G. Kranias, and I. Edes. 1994. Thyroid hormoneinduced alterations in phospholamban protein expression. Regulatory effects on sarcoplasmic reticulum $\mathrm{Ca}^{2+}$ transport and myocardial relaxation. Circ. Res. 75:245-251.

30. Lenihan, D. J., M. C. Gerson, B. D. Hoit, and R. A. Walsh. 1995. Mechanisms, diagnosis, and treatment of diastolic heart failure. Am. Heart J. 130: 153-156.

31. Garvey, J. L., E. G. Kranias, and R. J. Solaro. 1988. Phosphorylation of $\mathrm{C}$-protein, troponin I and phospholamban in isolated rabbit hearts. Biochem. J. 249:709-714.

32. Presti, C. F., L. R. Jones, and J. P. Lindemann. 1985. Isoproterenolinduced phosphorylation of a 15-kilodalton sarcolemmal protein in intact myocardium. J. Biol. Chem. 260:3860-3867.

33. Cory, C. R., R. W. Grange, and M. E. Houston. 1994. Role of sarcoplasmic reticulum in loss of load-sensitive relaxation in pressure overload cardiac hypertrophy. Am. J. Physiol. 266:H68-H78.

34. Rockman, H. A., S. Ono, R. S. Ross, L. R. Jones, M. Karimi, V. Bhargava, J. Ross, Jr., and K. R. Chien. 1994. Molecular and physiological alterations in murine ventricular dysfunction. Proc. Natl. Acad. Sci. USA. 91:26942698. 